\title{
INDUCTION CHEMOTHERAPY FOR T4 CENTRALLY LOCATED NON-SMALL CELL LUNG CANCER
}

Erino A. Rendina, MD

Federico Venuta, MD

Tiziano De Giacomo, MD

Anna Maria Ciccone, MD

Giovanni Ruvolo, MD

G. Furio Coloni, MD

Costante Ricci, MD

Sponsor: G. Alexander Patterson, MD
Objective: We used induction chemotherapy in a prospective, single-institution clinical trial intended to achieve resectability in patients with centrally located, unresectable T4 non-small cell lung cancer. Other types of IIIB disease were excluded. Methods: Between January 1990 and April 1996, we enrolled 57 patients with histologically confirmed non-small cell lung cancer. Eligibility criteria for T4 were clinical (superior vena cava syndrome, 9 patients), vocal cord paralysis (6 patients), dysphagia from esophageal involvement ( 1 patient), radiologic (computed tomography and magnetic resonance evidence of infiltration, 10 patients), bronchoscopic (tracheal infiltration, 11 patients), and thoracoscopic (histologically proven mediastinal infiltration, 20 patients). After 3 cycles of cisplatin $\left(120 \mathrm{mg} / \mathrm{m}^{2}\right)$, vinblastine $\left(4 \mathrm{mg} / \mathrm{m}^{2}\right)$, and mitomycin $\left(2 \mathrm{mg} / \mathrm{m}^{2}\right)$, patients were reevaluated. Results: Forty-two patients (73\%; 36 men, 6 women; age range, 42-75 years; mean, 58 years) responded to therapy and underwent thoracotomy; 11 patients did not respond, and 4 patients had major toxicity. Thirty-six patients (63\% of the entire group) had complete resection. We performed 4 exploratory thoracotomies, 6 pneumonectomies, 32 lobectomies ( 20 procedures were associated with reconstruction of hilar-mediastinal structures). Overall, 4 patients had no histologic evidence of disease. We had 2 bronchopleural fistulas with 1 death and 5 other major complications. Overall survival at 1 and 4 years is $61.4 \%$ and $19.5 \%$, respectively. Forty-two patients $(73 \%)$ underwent exploratory operation, with a 4-year survival of $25.9 \%$; 36 patients $(63 \%)$ had complete resection, with a 4-year survival of $30.5 \%$. Conclusions: Induction chemotherapy is effective for downstaging and surgical reconversion of centrally located T4 non-small cell lung cancer. Survival is promising, especially in patients whose disease becomes resectable. (J Thorac Cardiovasc Surg 1999;117:225-33)
In the late 1980s a number of studies showed that stage IIIA non-small cell lung cancer (NSCLC) could be downstaged and reconverted to surgical treatment by induction chemotherapy or chemoradiotherapy. ${ }^{1-3}$ It was also demonstrated that induction therapy followed by an

From the Department of Thoracic Surgery, University "La Sapienza," Rome, Italy.

Consulting Statistician: Dr. Claudio Ceccarelli, National Institute of Statistics (ISTAT).

Read at the Seventy-eighth Annual Meeting of The American Association for Thoracic Surgery, Boston, Mass, May 3-6, 1998.

Received for publication May 8, 1998; revisions requested June 30, 1998; revisions received Aug 14, 1998; accepted for publication Oct 1, 1998.

Address for reprints: Erino A. Rendina, MD, Department of Thoracic Surgery, University "La Sapienza” Rome, Policlinico Umberto I', 00161, Rome, Italy.

Copyright (C) 1999 by Mosby, Inc.

$0022-5223 / 99 \$ 8.00+0 \quad \mathbf{1 2 / 6 / 9 4 9 7 7}$ operation improved survival, especially in patients with ipsilateral mediastinal nodal metastasis (N2). ${ }^{4}$ After these promising results, the Southwest Oncology Group (SWOG) devised a phase II trial intended to clarify whether the benefits of induction therapy could be extended to patients with stage IIIB disease. ${ }^{5-7}$ Albain and colleagues ${ }^{6}$ concluded that there was no survival difference between stage IIIA and stage IIIB and reported an encouraging 24\% 3-year survival for the latter group. However, stage IIIB NSCLC entails either lesions that show a tendency towards distant spread (positive pleural effusion; N3 disease) or locally infiltrative tumors that involve by direct extension the mediastinal organs (T4 "mediastinum"). From a surgical point of view these latter tumors, which might be described as "T4 centrally located" lesions, are often technically unresectable. When a primary operation is attempted, the resection is rarely complete and the long-term survival is a discour- 
aging $8 \% .{ }^{8}$ More than one half of the patients experience local recurrence, and approximately $40 \%$ of the patients have distant metastasis. ${ }^{9,10}$ Complete resection remains, nevertheless, the mainstay in the treatment of NSCLC; and with the enhancement offered by induction therapy, it has been demonstrated in other settings that more complete resections can be achieved. Also, systemic treatment may prevent or reduce the development of distant metastasis. On the basis of the optimistic results of the group of Memorial Sloan Kettering Cancer Center ${ }^{4}$ with induction chemotherapy for $\mathrm{N} 2$ disease, we were attracted by the possibility of reconverting the subgroup of T4 unresectable lesions to operation. We therefore used induction chemotherapy in a prospective clinical trial intended to achieve resectability in patients with centrally located T4 NSCLC.

\section{Materials and methods}

Between January 1990 and April 1996, we enrolled 57 patients with histologically confirmed NSCLC in a prospective single-institution clinical trial intended to evaluate the efficacy of induction chemotherapy followed by an operation. Only patients with centrally located T4 tumors were included independently from the $\mathrm{N}$ status. All other types of stage IIIB (cytologically positive pleural effusion; contralateral mediastinal or supraclavicular lymph node metastasis) and those patients with distant metastasis were excluded. Pretreatment evaluation included clinical history, physical examination, complete laboratory work-up, chest radiography, electrocardiography, pulmonary function tests, total body computed tomography (CT), and bronchoscopy. Bone scans and magnetic resonance (MR) were selectively used. The eligibility criteria for $\mathrm{T} 4$ were as follows:

1. Clinical: superior vena cava (SVC) syndrome (9 patients), vocal cord paralysis (6 patients), dysphagia from esophageal involvement (1 patient).

2. Radiologic: CT and MR evidence of infiltration (10 patients). This group was subjected to a potential margin of error. We were conscious of this risk and had made all efforts to restrict the number of these patients. Because it is the rule in our Department, all CT and MR images were reviewed by us before a final decision was reached. In 2 patients the tumor surrounded the SVC for more than $50 \%$ of its circumference without causing SVC syndrome; 2 patients had erosion of the vertebral body; 3 patients experienced the sudden appearance of atrial fibrillation associated with a neoplasm occluding the lower pulmonary vein and extending over the left atrium with no cleavage plane; in 3 patients the tumor infiltrated the aorta and the origin of the left pulmonary artery inside the pericardium. Eight of these patients also had mediastinoscopy.

3. Bronchoscopic: Infiltration of the trachea, tracheobronchial angle, or carina (11 patients).

4. Thoracoscopic: Histologically proven mediastinal infiltration (20 patients). Thoracoscopy was performed in these patients as previously described. ${ }^{11,12}$
All patients fulfilling these criteria and undergoing induction chemotherapy at our institution between January 1990 and April 1996 were included in the study.

We did not use thoracotomy as a staging technique. Mediastinoscopy was performed in 38 patients. Four patients underwent mediastinoscopy and thoracoscopy. All invasive staging procedures (bronchoscopy, mediastinoscopy, and thoracoscopy) were performed by us. The standard chemotherapy protocol consisted of 3 cycles of the mitomycinvinblastine-platinum regimen. Cisplatin $\left(120 \mathrm{mg} / \mathrm{m}^{2}\right)$ was administered intravenously in a 20-minute infusion with hydration on days 1 and 29 . Mitomycin $\left(2 \mathrm{mg} / \mathrm{m}^{2}\right)$ was administered intravenously on days 1 to 5 . Vinblastine (4 $\mathrm{mg} / \mathrm{m}^{2}$ ) was administered on days $1,8,15,22$, and 29 . One to 3 weeks after completion of the induction chemotherapy, the disease of each patient was completely reevaluated. The disease was restaged by clinical examination, complete laboratory work-up, chest radiography, total body $\mathrm{CT}$, and bone scan. The cardiorespiratory status of the patient was reassessed by spirometry, blood gas analysis, and electrocardiography. Echocardiography, ventilation/perfusion lung scans, Holter monitoring, and stress testing were selectively performed in patients who were at risk. Patients in whom distant metastasis developed (5 patients), those who showed local progression of disease (6 patients), and those having severe toxicity related to the induction therapy (4 patients) were not considered for an operation. All surgical procedures were performed by the same team. Standard posterolateral thoracotomy, pulmonary resection, and complete hilar and mediastinal lymphadenectomy were attempted. The resection of portions of the infiltrated mediastinal organs was performed when necessary to obtain tumor-negative resection margins. Multiple frozen section examinations were performed routinely. The resection was recorded as complete when all resection margins were negative. After the operation, patients with complete resection of the primary tumor, with microscopically negative margins, and negative $\mathrm{N} 1$ and $\mathrm{N} 2$ nodes did not receive additional treatment. Patients whose disease appeared unresectable or who had microscopically positive margins or positive $\mathrm{N} 1$ and/or $\mathrm{N} 2$ nodes received additional chemotherapy (2 cycles of the same regimen), radiotherapy (3.500 rad), or chemoradiotherapy. Postoperative adjuvant therapy was started 3 to 6 weeks after the operation. Follow-up is complete for all patients. Survival is reported by intent to treat and was calculated from the start of induction chemotherapy with the product limit method by Kaplan and Meier. ${ }^{13}$ Differences among curves were analyzed by the log-rank test and the chi-squared test. Completeness of resection, preinduction therapy $\mathrm{N}$ status, and histologic type were evaluated as predictors of survival. All deaths were included in the analysis.

\section{Results}

From January 1990 to April 1996, 57 patients were entered in the study. There were 47 men and 10 women with a median age of 59 years (range, $42-75$ years). Squamous cell carcinoma was the most common cell 
Table I. Staging before induction therapy (57 patients) and at thoracotomy (42 patients)

\begin{tabular}{|c|c|c|}
\hline & $\begin{array}{c}\text { Before } \\
\text { induction therapy }\end{array}$ & Thoracotomy \\
\hline \multicolumn{3}{|c|}{ Stage IIIB (n=61) } \\
\hline T4 N2 & 34 & 4 \\
\hline T4 N1 & 6 & \\
\hline T4 N0 & 17 & \\
\hline \multicolumn{3}{|c|}{ Stage IIIA ( $(n=13)$} \\
\hline T3 N2 & & 2 \\
\hline T3 N1 & & 2 \\
\hline T3 N0 & & 2 \\
\hline $\mathrm{T} 2 \mathrm{~N} 2$ & & 6 \\
\hline T1 N2 & & 1 \\
\hline \multicolumn{3}{|c|}{ Stage II $(n=13)$} \\
\hline $\mathrm{T} 2 \mathrm{~N} 1$ & & 12 \\
\hline T0 N1 & & 1 \\
\hline \multicolumn{3}{|c|}{ Stage I $(\mathrm{n}=8)$} \\
\hline T2 N0 & & 6 \\
\hline T1 N0 & & 2 \\
\hline \multicolumn{3}{|c|}{ Stage $0(n=4)$} \\
\hline TOTAL & 57 & 42 \\
\hline
\end{tabular}

type and was found in 31 patients (54\%). Eighteen patients $(31 \%)$ had adenocarcinoma, 6 patients (10\%) had large cell carcinoma, and 2 patients (3\%) had adenosquamous tumors. The invasive staging procedures (thoracoscopy, mediastinoscopy) were performed by us without complications. Of the 38 patients undergoing mediastinoscopy, 26 patients had N2 disease. Seventeen patients had only 2 of the scheduled 3 cycles, and in 13 patients the dose and timing of the administration of the drugs had to be reduced or modified. The preinduction and postoperation staging is reported in Table I. The list of infiltrated organs is reported in Table II. Induction therapy was generally well tolerated. The most common toxic effects were similar to those reported in other studies that used the MVP regimen. ${ }^{13}$ Four patients experienced major toxicities that precluded an operation. Only those 4 patients had severe toxicity; there were no other patients who had induction chemotherapy and were removed before being included in the group of 57 patients. Nephrotoxicity caused by cisplatin occurred in 2 patients; severe neutropenia associated with recurrent infection occurred in 1 patient, and severe mitomycininduced interstitial lung disease developed in 1 patient. Eleven patients did not respond to induction therapy; 6 of those patients had adenocarcinoma (54\%); 4 patients had squamous cell carcinoma, and 1 patient had large cell carcinoma. Four patients had persistent biopsy-proven airway disease; distant metastasis developed in 5 patients, and 2 patients displayed local progression of disease. Forty-two patients (73\% of the entire study group; 36 men and 6 women; age range, $42-75$ years; median, 58
Table II. Mediastinal organs infiltrated by tumor in 57 patients

\begin{tabular}{lc}
\hline Organ & No. of patients \\
\hline Trachea & 13 \\
SVC & 13 \\
Esophagus & 3 \\
Atrium & 3 \\
Extensive mediastinal infiltration & 13 \\
Spine & 3 \\
Recurrent laryngeal nerve & 6 \\
Pulmonary artery & 8 \\
Aorta & 5 \\
Azygos vein & 2 \\
\hline
\end{tabular}

years) responded to therapy and underwent thoracotomy. Patients undergoing operation showed stability or regression of tumor on CT. Of the 20 patients who had histologic evidence of $\mathrm{N} 2$ before induction and who underwent an operation, 2 patients $(10 \%)$ were converted to N0, and 6 patients (30\%) were downstaged to N1. No patient $\mathrm{N} 0$ at preinduction staging was found to be $\mathrm{N} 2$ after the operation; however, 7 patients who were mediastinoscopy negative turned out to have $\mathrm{N} 1$ disease at the time of the operation. Of 13 patients with central airway invasion, 6 patients did not respond to induction therapy; 1 patient had chemotherapy-induced toxicity, and 6 patients underwent an operation. Of these, 1 patient had incomplete resection, and 5 patients had complete resection. The surgical procedure was almost constantly complicated by fibrosis and obliteration of tissue planes; the tumor burden was often surrounded by a reactive growth involving the previously infiltrated organs. The extensive use of frozen sections proved crucial to avoid the unnecessary resection of mediastinal organs that seemed macroscopically involved. Thirty-six patients $(63 \%$ of the initial study group and $85 \%$ of those undergoing operations) had complete resection. We performed 4 exploratory thoracotomies, 6 pneumonectomies ( 1 incomplete resection), and 32 lobectomies (1 incomplete resection). Twenty procedures were associated with resection and reconstruction of hilar and mediastinal organs. The details of the surgical procedures are reported in Table III. Six of 8 patients who were staged as T4 by CT and MR had extended and complex operations (Table IV). In 3 patients the resection of the SVC was required. The vein was reconstructed by a patch of autologous pericardium in 2 patients and by an armored polytetrafluoroethylene tube in 1 patient. Resection and reconstruction of the bronchus and/or pulmonary artery was performed in 14 patients, with the technique previously described. ${ }^{14}$ Pathologic staging is reported in Table I. In 4 patients (7\% of the entire group and $9.5 \%$ of patients who had 
Table III. Surgical procedures in 42 patients

\begin{tabular}{lc}
\hline Surgical procedure & No. of patients \\
\hline Exploratory thoracotomy & 4 \\
Pneumonectomy & 3 \\
$\quad$ Standard & 2 \\
Intrapericardial & 1 \\
Resection of atrium & \\
Lobectomy/bilobectomy & 15 \\
Standard & 3 \\
Reconstruction of SVC & 14 \\
Pulmonary artery or bronchial sleeve & 42 \\
TOTAL & \\
\hline
\end{tabular}

undergone thoracotomy), no histologic evidence of tumor was found. Major complications occurred in 7 patients (16\%), 1 of whom died (2.3\%). We had 2 bronchopleural fistulas ( 1 death), 1 acute myocardial infarction, 2 cases of severe respiratory insufficiency, 1 empyema, and 2 wound infections. Nine additional patients experienced delayed lung reexpansion and prolonged air leaks. Chest tube duration ranged between 4 and 17 days, excluding the patients with bronchopleural fistula and empyema. Postoperative adjuvant therapy was scheduled for patients having incomplete resection and for those with $\mathrm{N} 1$ or N2 disease. However, of 6 patients who had incomplete resection, 1 patient died of bronchopleural fistula and 2 patients were too compromised to withstand further therapy and died 6 months after the operation with local recurrence. Follow-up is complete for all patients; 17 to 79 months (mean, 29 months) after the start of induction therapy, 36 patients have died of cancer (18 patients, of metastasis; 11 patients, of local recurrence; 4 patients, of neoplastic cachexia; and 3 patients, of metastasis plus local recurrence); 3 patients died of treatment-related causes (chemotherapy related toxicity, bronchopleural fistula, acute myocardial infarction 1 month after the operation); 3 patients died of unrelated causes; 2 patients are alive with disease; 13 patients are alive and free of disease. With a $95 \%$ confidence limit, survival for all patients is $61.4 \%(48.9 \%-73.9 \%)$ at 1 year and $19.8 \%$ (8.3\%-31.3\%) at 4 years. Four-year survival was $25.9 \%$ for the 42 patients who underwent an operation and $30.4 \%$ for the 36 patients having complete resection (Fig 1). Completeness of resection $(P=.0075)$ and pretreatment $\mathrm{N}$ status $(P=.00009 ;$ Fig 2$)$ significantly influenced survival. Histology did not appear to influence survival $(P$ $=.20$ ).

\section{Comment}

The prognosis of NSCLC with mediastinal invasion is uniformly poor. Complete resection is feasible in only
Table IV. Surgical procedures in 8 patients staged T4 by CT or MR imaging

\begin{tabular}{lc}
\hline Procedure & No. of patients \\
\hline SVC reconstruction & 1 \\
Extrapleural resection and shaving & 1 \\
$\quad$ of the vertebral body & \\
Resection of the atrium & 1 \\
Intrapericardial pneumonectomy & 1 \\
Bronchial sleeve resection with implantation & 1 \\
$\quad$ of the bronchus intermedius at the carina & \\
Sleeve resection of the pulmonary artery & 1 \\
Standard lobectomy & 2 \\
ToTAL & 8 \\
\hline
\end{tabular}

$22 \%$ to $60 \%$, and the overall reported 5-year survival is around $9 \% .{ }^{15}$ Within this group, T3N0 lesions have a more favorable outcome, but still the complete resection rate does not exceed $66 \% .{ }^{8}$ On the other hand, T3N1 or $\mathrm{N} 2$ tumors and $\mathrm{T} 4$ lesions are resectable in no more than $20 \%$ of the cases, and their 5-year survival is a disappointing $8 \%{ }^{8}{ }^{89}$ Radiation therapy does not seem to be more active with T4 lesions; a study from Shields and coworkers ${ }^{16}$ showed that, although $26 \%$ of the tumors were sterilized by radiation therapy, this had no effect on survival. More recently, the use of hyperfractionated very high-dose (>6000 cGy) radiotherapy could not enhance survival to more than $29 \%$ at 2 years. ${ }^{17}$ All in all, the prognosis of patients with T4 NSCLC involving the mediastinum is dismal, and at the present time the appropriate treatment has not been found.

Because induction therapy based on the MVP regimen proved effective in downstaging $\mathrm{N} 2$ disease, we have used it for unresectable T4 tumors. An induction program of concurrent chemoradiotherapy could theoretically seem more appropriate for patients whose major problem is a locally infiltrative, often large, tumor burden associated with the threat of distant metastases in $40 \%$ of cases. In this setting, however, radiation therapy should be administered at high doses and through extended mediastinal and pulmonary fields. Our preceding experience with preoperative radiotherapy for hilomediastinal NSCLC and its ensuing postoperation complications helped us to avoid radiation therapy; our perplexities were also confirmed by Fowler and associates ${ }^{18}$ and by Rush and Benfield ${ }^{19}$ who underscored the risk of chemoradiotherapy in a preoperative setting. On the other hand, the toxicity, response rate, resectability, and survival in our study are comparable to those reported in the SWOG trial ${ }^{6,7}$ in which chemoradiotherapy was used. We reduced the cumulative doses of the drugs in 30 patients, but the remaining 27 patients tolerated the full regimen well. 


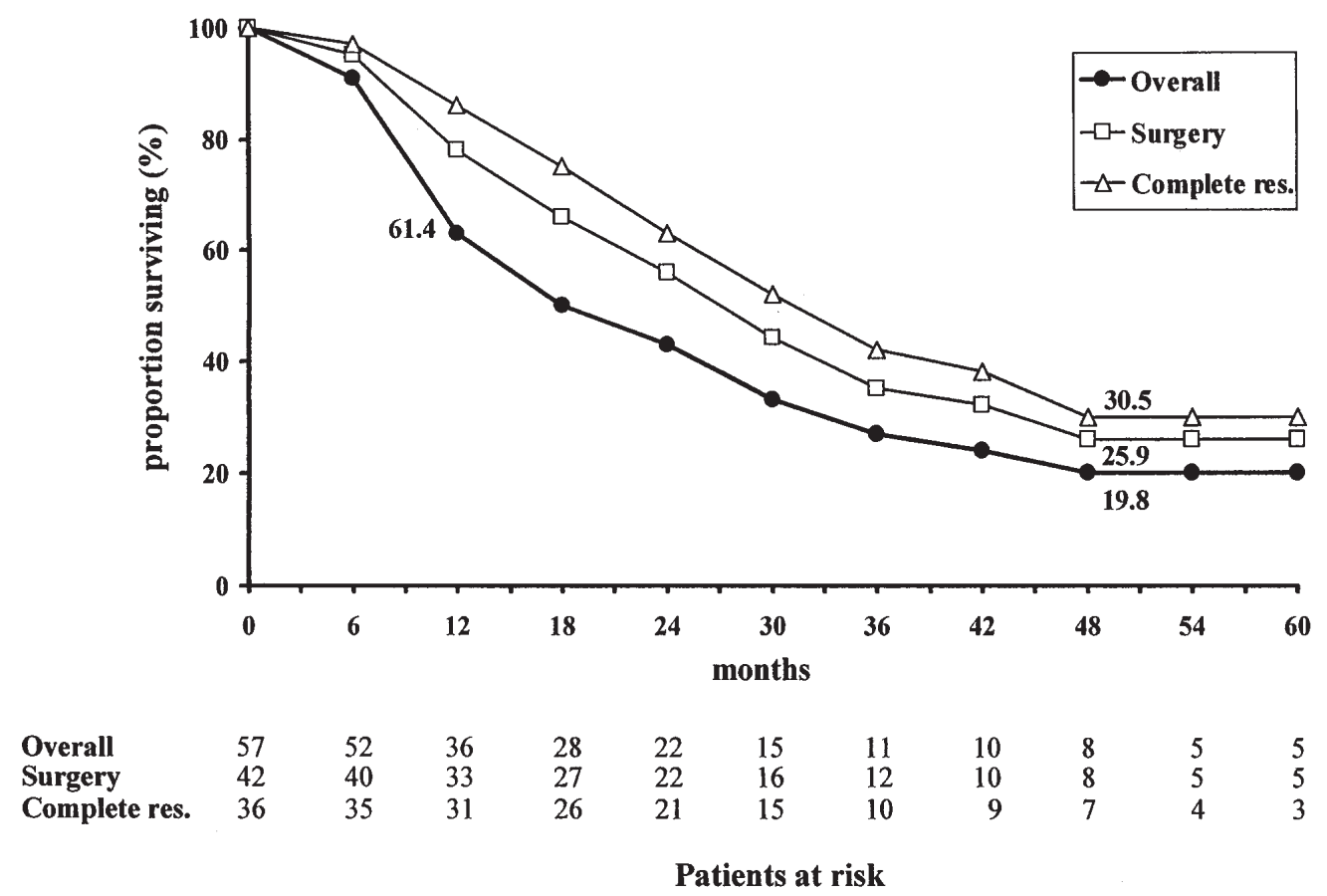

Fig 1. Cumulative survival of T4 NSCLC undergoing induction chemotherapy: 57 patients, 1990 to 1996. Survival is calculated for all 57 patients, for those undergoing surgery (42 patients), and for those having complete surgical resection (36 patients).

Our recommendation for the current clinical practice, before the efficacy of new protocols is proved, is therefore to judiciously use the MVP regimen. Outside of clinical trials, however, this regimen can be used with more flexibility concerning the doses and timing of administration.

Previous studies have grouped all stage IIIB tumors on the basis of their known poor prognosis and because this category of the disease of these patients is uniformly considered inoperable or unresectable. In this case, "inoperable" suggests the concept of systemic tumor involvement (eg, N3), and "unresectable" indicates simply the technical impossibility to remove the neoplasm. We elected to perform an operation in only those patients with locally infiltrative T4 tumors because this presentation of disease represents mainly an operative problem. In fact, the tumor is often unresectable, but (at least temporarily) it tends to expand locally without systemic spread. ${ }^{8,10}$ This clinical behavior may recall the attitude of T3 tumors involving the chest wall and thus further underscore the importance of complete resection. In selected patients with $\mathrm{T} 4$ tumors that involve the carina, surgical resection is indeed the treatment of choice. ${ }^{20}$ Also, occasional patients have been treated successfully by the resection of mediastinal organs, especially the SVC, ${ }^{21-23}$ aortic arch, ${ }^{21}$ vertebral column, ${ }^{24}$ and main pulmonary artery. ${ }^{25}$ Still, a few patients can benefit from these procedures, mainly because of the technical impossibility to do so. Our goal was to explore the possibility of converting unresectable $\mathrm{T} 4$ tumors into resectable tumors and record the outcome of the tumors in these patients.

Accurate staging before induction therapy is crucial to discriminate between T3 and T4. ${ }^{26}$ Clinical and bronchoscopic confirmation of $\mathrm{T} 4$ is unquestionable and uniformly accepted ${ }^{6,7}$ although CT and MR imaging, mediastinoscopy, thoracoscopy, and thoracotomy each have pros and cons that require special consideration. Radiologic imaging is associated with a potential margin of error. We have studied mediastinal infiltration by CT with intraoperative controls since $1981^{27}$ and have acquired a large direct experience in the field. Ten patients in our group were staged radiologically: one patient did not undergo an operation for tumor progression, and another patient did not undergo an operation because of toxicity. For the 8 patients (19\% of those undergoing operation) who underwent an operation, Table IV demonstrates that signs of previous or permanent infiltration of the mediastinum were actually present at the operation. Also, 8 of 10 patients underwent 


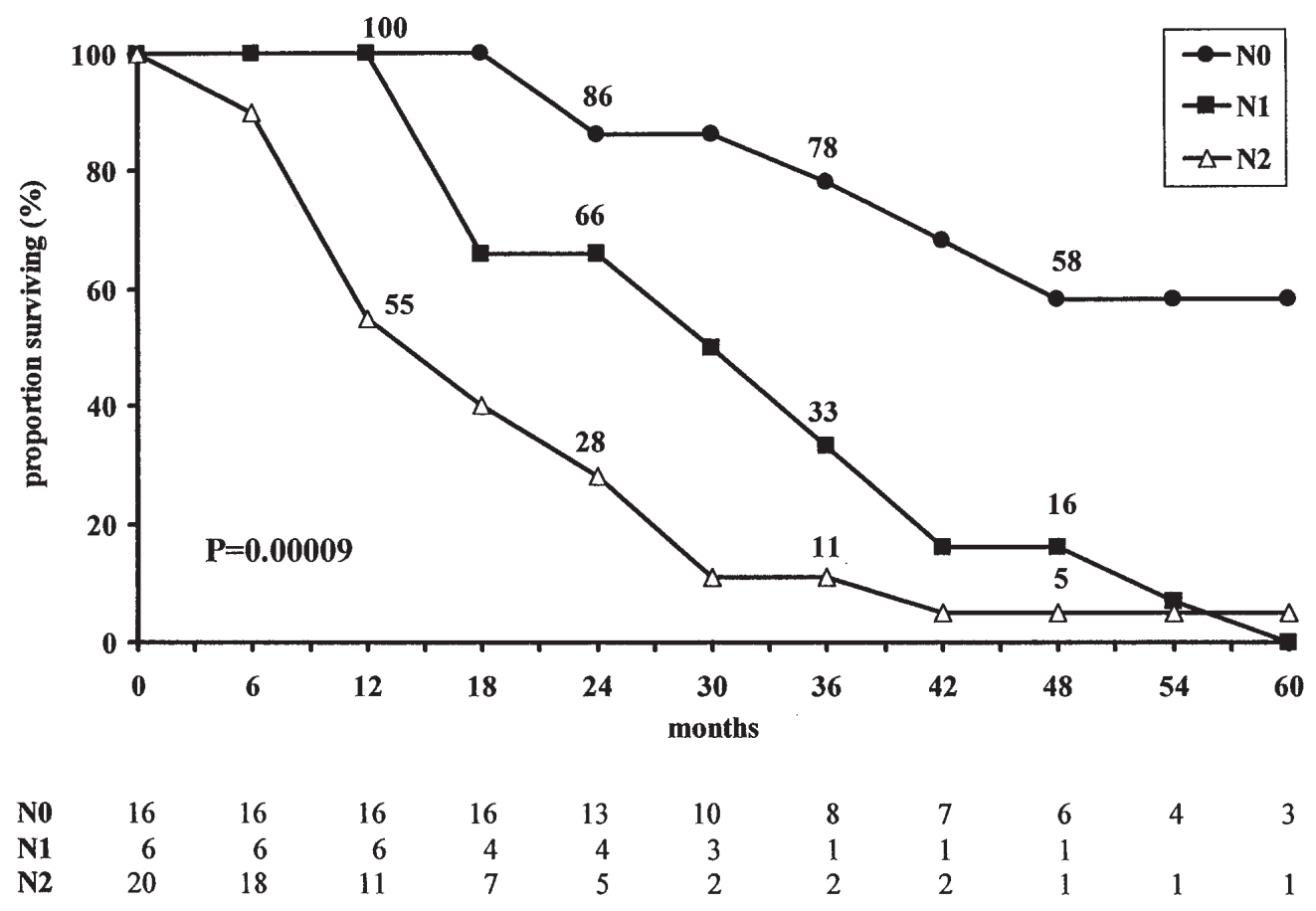

Patients at risk

Fig 2. Cumulative survival of T4 NSCLC undergoing induction and surgery: 42 patients, 1990 to 1996. Survival differences after lymph node status. Survival is calculated for patients with N2 disease (20 patients), for patients with N1 disease (6 patients), and for patients with N0 disease (16 patients).

mediastinoscopy, which proved positive in 4 patients. The latter approach was accepted as a T4 staging method in other studies ${ }^{6,7}$; however, mediastinoscopy might be a source of incertitude as well, because it can hardly discriminate between extracapsular N2 and primary tumor infiltration. Thoracoscopy might also suffer to some extent from interoperator discrepancies because of the limited view field, the impossibility to directly palpate the tissues, and the risk of dissecting the lung and mediastinum. Although we were satisfied with the use of thoracoscopy in this setting, ${ }^{12}$ theoretically some tumors could be downstaged to $\mathrm{T} 3$ by a more aggressive (though dangerous) dissection. In this study, we have made all efforts to be rigorous with the staging. Therefore CT imaging was accepted only in selected cases; otherwise thoracoscopy was performed. On the basis of our experience, we would now suggest that, in experienced hands, CT could be more often accepted for the diagnosis of T4. As far as thoracotomy is concerned, we do not consider it acceptable as a staging method. Furthermore, if positive evidence of T4 must be acquired by this technique, that should occur only after thoracoscopy proved inconclusive, thus demonstrating that the case is a particularly difficult one. The surgeon is therefore expected to accept for his or her patient and for him or herself a long-lasting, difficult, and dangerous operation.

Overall, the staging of $\mathrm{T} 4$ does not benefit at the present time from precise guidelines; we have to recognize that there is no such specific technique available, such as mediastinoscopy, which exists for N2. If clinical experimentation in this field has to be pursued, precise staging guidelines should be developed that take into account accuracy and patient safety.

Toxicity, morbidity, and death related to the protocol do not differ from what has been extensively reported for chemotherapy-alone induction protocols. ${ }^{3,4} \mathrm{We}$ reduced the total dose of mitomycin in 23 patients. One patient was removed from the study because of mitomycin-induced toxicity, and one patient, who is alive and free from tumor 50 months after the operation, has a stabilized form of interstitial lung disease. Our toxicity-related mortality rate of $7 \%$ does not exceed that reported in studies on chemoradiotherapy $(10 \%) .{ }^{6,7}$ Also, the complete resection rate in our study is in line with the reports of Faber and associates ${ }^{2}(85 \%)$, 
Martini and colleagues ${ }^{4}$ (65\%), and Rush and cowork$\operatorname{ers}^{7}(83 \%)$. We want to point out, however, that most tumors in our series were technically unresectable before induction therapy, and this furthermore emphasizes the efficacy of preoperative chemotherapy.

Surgical difficulties after induction chemotherapy have been uniformly reported. In more than $50 \%$ of our patients who underwent resection, the procedure was complicated by the excision of hilar or mediastinal organs; in the remaining cases, a standard lobectomy or pneumonectomy could be carried out only after accurate and difficult dissection. It seems that in our series more reconstructive and "complex" procedures occurred in comparison with other studies. First, we tend to attribute this fact to our preference for bronchovascular reconstructive procedures as opposed to pneumonectomy. Also, we had the impression that lesser resections, such as sleeve lobectomy, could be accomplished because of induction therapy. Second, most studies on induction chemotherapy were based on N2 lesions, which may be not so locally infiltrative as $\mathrm{T} 4$. There is general agreement that the lung parenchyma should be spared at all costs and that the clinical course of these patients is often difficult. We believe that surgical judgment and perioperative care rather than technical ability is the key factor in this setting. Of 23 patients who died of cancer after an operation, 14 patients $(60 \%)$ had distant metastases and only 7 patients $(30 \%)$ had local recurrence. We interpret this data as a confirmation of the efficacy of induction chemotherapy in the local control of NSCLC. Survival is promising for the entire surgical group but still disappointing for patients either whose disease remained inoperable or who had incomplete resection or N2 disease. However, in the N2 group some patients survived more than 3 or 4 years; we infer that if more effective chemotherapy regimens will become available, probably also this subset could benefit from resection. Central airway involvement is an interesting feature within the T4 stage, because it can be easily and reliably monitored noninvasively. About one half of our patients did not respond to the induction regimen, and the other half had an operation. Two of the patients in the latter group are free of disease after 18 and 34 months. This suggests that although the overall outcome is not optimistic, downstaging of central airway disease can be achieved and cure is possible.

We conclude that, in patients with T4 lesions who underwent induction chemotherapy, it is reasonable to attempt resection. The complete resectability rate in our group was $63 \%$ (including patients who did not undergo an operation), a rate significantly higher than that reported in patients treated by primary surgery. ${ }^{8-10}$ Four patients had major toxicity from the induction regimen (7\%), and 3 patients died of treatment-related causes $(5.2 \%)$. Four-year survival is $19.5 \%$ for the entire group and $25.9 \%$ for those undergoing an operation. Our data indicate that induction chemotherapy is effective for downstaging and surgical reconversion of centrally located, unresectable T4 NSCLC. Toxicity, morbidity, and mortality rates are acceptable; survival is promising, but it remains to be confirmed in larger series of patients and phase III trials.

\section{REFERENCES}

1. Martini N, Kris MG, Gralla RJ, et al. The effect of preoperative chemotherapy on the resectability on non-small cell lung carcinoma with mediastinal lymph node metastases (N2 M0). Ann Thorac Surg 1988;45:370-9.

2. Faber LP, Kittle FC, Warren WH, et al. Preoperative chemotherapy and irradiation for stage III non-small cell lung cancer. Ann Thorac Surg 1989;47:669-77.

3. Burkes RL, Ginsberg RJ, Shepherd FA, et al. Induction chemotherapy with mitomycin, vindesine and cisplatin for stage III unresectable non-small cell lung cancer: results of the Toronto phase II trial. J Clin Oncol 1992;10:580-6.

4. Martini N, Kris MG, Flehinger BJ, et al. Preoperative chemotherapy for stage IIIA (N2) lung cancer: the Sloan-Kettering experience with 136 patients. Ann Thorac Surg 1993;55:1365-74.

5. Rusch VW, Albain KS, Crowley JJ, et al. Surgical resection of stage IIIA and stage IIIB non-small-cell lung cancer after concurrent induction chemoradiotherapy: a Southwest Oncology Group trial. J Thorac Cardiovasc Surg 1993;105:97-106.

6. Albain KS, Rusch VW, Crowley JJ, et al. Concurrent cisplatin/ etoposide plus chest radiotherapy followed by surgery for stages IIIA (N2) and IIIB non-small-cell lung cancer: mature results of Southwest Oncology Group Phase II study 8805. J Clin Oncol 1995;13:1880-92.

7. Rush VW, Albain KS, Crowley JJ, et al. Neoadjuvant therapy: a novel and effective treatment for stage IIIB non-small cell lung cancer. Ann Thorac Surg 1994;58:290-5.

8. Martini N, Yellin A, Ginsberg RJ, et al. Management of non-small cell lung cancer direct mediastinal involvement. Ann Thorac Surg 1994;58:1447-51.

9. Naruke T, Goya T, Tsuchiya R, Suemasu K. Prognosis and survival in resected lung carcinoma based on the new international staging system. J Thorac Cardiovasc Surg 1988;96:440-7.

10. Van Raemdonck DE, Schneider A, Ginsberg RJ. Surgical treatment for higher stage non-small cell lung cancer. Ann Thorac Surg 1992;54:999-1013.

11. Rendina EA, Venuta F, De Giacomo T, et al. Comparative merits of thoracoscopy, mediastinoscopy and mediastinotomy for mediastinal biopsy. Ann Thorac Surg 1994;57:992-5.

12. De Giacomo T, Rendina EA, Venuta F, Della Rocca G, Ricci C. Thoracoscopic staging of non-small cell lung cancer before neoadjuvant therapy. Ann Thorac Surg 1997;64:1409-11.

13. Kaplan EL, Meyer P. Nonparametric estimation from incomplete observations. J Am Stat Assoc 1958;53:457-81.

14. Rendina EA, Venuta F, De Giacomo T, et al. Safety and efficacy of bronchovascular reconstruction after induction chemotherapy for lung cancer. J Thorac Cardiovasc Surg 1997;114:830-7. 
15. Burt ME, Pomerantz AH, Bains MS. Results of surgical treatment of stage III lung cancer invading the mediastinum. Surg Clin North Am 1987;67:987-1000.

16. Shields TW, Higgins GA, Lawton R, et al. Preoperative x-ray therapy as an adjuvant in the treatment of bronchogenic carcinoma. J Thorac Cardiovasc Surg 1970;59:49-61.

17. Cox JD, Azarnia N, Byhardt RW, et al. A randomized phase I/II trial of hyperfractionated radiation therapy with total doses of 60.0 Gy to 79.2 Gy: possible survival benefit with $69.9 \mathrm{~Gy}$ in favorable patients with Radiation Therapy Oncology Group stage III non-small cell lung carcinoma: report of Radiation Therapy Oncology Group 83-11. J Clin Oncol 1990;8:1543-55.

18. Fowler WC, Langer CJ, Curran WJ Jr, Keller SM. Postoperative complications after combined neoadjuvant treatment of lung cancer. Ann Thorac Surg 1993;55:986-9.

19. Rush VW, Benfield JR. Neoadjuvant therapy for lung cancer: a note of caution. Ann Thorac Surg 1993;55:820-1.

20. Tsuchiya R, Goya T, Naruke T, Suemasu K. Resection of tracheal carina for lung cancer: procedure, complications, and mortality. J Thorac Cardiovasc Surg 1990;99:779-87.

21. Nakahara K, Ohno K, Mastumura A, Ogawa J, Inoue H. Exended operation for lung cancer invading the aortic arch and superior vena cava. J Thorac Cardiovasc Surg 1989;97:428-33.

22. Inoue $H$, Shohtsu A, Koide $S$, et al. Resection of the superior vena cava for primary lung cancer: 5 years' survival. Ann Thorac Surg 1990;50:661-2.

23. Dartevelle P, Chapelier A, Navajas M, et al. Replacement of the superior vena cava with polytetrafluoroethylene grafts combined with resection of mediastinal-pulmonary malignant tumors: report of thirteen cases. J Thorac Cardiovasc Surg 1987;94:361-6.

24. De Meester TR, Albertucci M, Dawson PJ, Montner SM. Managment of tumor adherent to the vertebral column. J Thorac Cardiovasc Surg 1989;97:373-8.

25. Ricci C, Rendina EA, Venuta F, et al. Reconstruction of the pulmonary artery in patients with lung cancer. Ann Thorac Surg 1994;57:627-33.

26. Faber LP. In discussion of: Rush VW, Albain KS, Crowley JJ, et al. Neoadjuvant therapy: a novel and effective treatment for stage IIIB Non-Small Cell Lung Cancer. Ann Thorac Surg 1994; 58:290-5.

27. Rendina EA, Bognolo DA, Mineo TC, et al. Computed tomography for the evaluation of intrathoracic invasion by lung cancer. J Thorac Cardiovasc Surg 1987;94:57-63.

\section{Discussion}

Dr L. Penfield Faber (Chicago, Ill). I would like to compliment Dr Rendina and his group on undertaking the treatment of this group of patients with clinically advanced lung cancer. These patients are extremely difficult to treat and achieve good therapeutic results.

I would like to caution those in the audience who have just heard this program of neoadjuvant chemotherapy, that it is a rigorous treatment protocol. One hundred and twenty milligrams of cisplatin per meter square can be difficult for the patient to tolerate the total dose, and mitomycin is associated with an increased incidence of pulmonary fibrosis and pulmonary complications after a surgical procedure. It is noted in your series that 30 patients had their total dosages modified and that a second group of patients received only 2 cycles with a decrease in the amount of drugs given. With your reported modifications, would you now recommend the same drug protocol, or due to toxicity associated with this vigorous program, would you now modify the recommended drug dosages?

It is noted that this neoadjuvant program has sterilized 7\% of the tumors that were resected. Previous reports of neoadjuvant programs that use chemotherapy in combination with radiation report a sterilization rate of $18 \%$ to $20 \%$, although the tumors may not be as clinically advanced as those in your series. Would this decreased incidence of tumor sterilization in the resected specimens alter your thinking concerning the use of radiation in combination with chemotherapy in the treatment of this group of patients?

Staging was accomplished by thoracoscopy in 20 patients, and I would question its use in clinical staging. It requires an additional anesthetic and procedure and may well disturb mediastinal tissue planes with possible seeding of tumor from biopsy sites. It was stated in the article that your group has had a great deal of experience with the CT scan and MR imaging in staging, and with the addition of the PET scan and mediastinoscopy, precise clinical staging could be accomplished without thoracoscopy. Would you recommend continuation of thoracoscopic clinical staging?

Another question relates to the concept of downstaging T4 disease. In other words, if there is viable tumor in the mainstem bronchus, can you convert a probable pneumonectomy to a standard lobectomy or sleeve lobectomy by neoadjuvant chemotherapy? Down staging also relates to mediastinal lymph nodes. In your series, did you convert any positive N2 lymph nodes to N0, as determined by surgical/pathological staging? This finding would further substantiate the efficacy of your neoadjuvant chemotherapeutic protocol.

The described postoperative adjuvant therapeutic program had variability, and specific indications were not provided in the article. Some patients with positive margins and/or positive nodes received radiation; some received chemotherapy, and some received both. Could you provide us with specific recommendations for postoperative adjuvant therapy, and is this type of therapy associated with any toxic manifestations?

The survival curve indicates that the patients with N0 disease had very good survival, while those with N1 and N2 disease did not do as well. With the poor results achieved in the group of patients with T4 N2 disease, would you now recommend routine mediastinoscopy in all patients submitted to this protocol? What is your definitive recommendation in a patient clinically staged with a T4 N2 disease? Should such a patient be submitted to neoadjuvant therapy followed by attempted surgical resection?

Dr Rendina. Indeed what you pointed out about the toxicity of our induction therapy regimen is true. Over one half of our patients had to undergo a modification of the chemotherapy regimen despite the absence of radiotherapy in the protocol. Two of the 4 patients who could not undergo an operation because of the toxic side effects of chemotherapy had nephrotoxicity. One other patient had multiple infections associated with neutropenia, and in the fourth patient intersti- 
tial lung disease occurred. We closed our study in 1996 also because the MVP regimen that we had been using was probably no more the best option for our patients. A protocol including platinum and gemcitabine would probably be more effective and less toxic, and we have it currently under investigation at our institution.

As far as sterilization of tumor is concerned, its rate is indeed lower in our group of patients than that obtained by other regimens. However, the sterilization rate per se does not necessarily translate into an increased complete resection rate and better long-term survival for the whole study group. What probably is more meaningful is the $63 \%$ overall complete resection rate that we report; these data parallel the $85 \%$ complete resection rate in the group of patients who underwent an operation. We believe this is the data to look at, especially in the setting of direct mediastinal tumor infiltration.

Staging is a very important issue in this setting, and we think that $\mathrm{CT}$ and MR imaging are dependable in many cases. Since 1981 we review in our department all CT images of our patients with lung cancer, comparing the images accurately with the intraoperative findings. With all due respect to the radiologists, I think that this probably gives us a better idea of the cleavage planes and tumor infiltration pattern, thus allowing for better interpretation of successive cases. Twenty patients underwent staging by video-assisted thoracoscopy. I agree with you that this is a questionable choice, and we have commented on our reasons for doing so in our article. All in all, we have used VATS in those cases where we were not $100 \%$ sure of the CT imaging, and retrospectively we found out that CT was very accurate in many patients. If clinical studies have to be pursued in the field of infiltrative, centrally located NSCLC, there is no doubt that one important point would be to establish precise staging guidelines to be followed by all investigators.

In our group, 38 patients underwent mediastinoscopy, and in another 7 patients evidence of N2 disease was acquired by VATS. Mediastinoscopy is an excellent tool for the staging of N2 disease, but I would not say the same for T4. It is impossible by mediastinoscopy to discriminate between extracapsular nodal infiltration and infiltration by direct extension of the primary lung tumor. PET scanning seems promising in this setting, but it is not yet available at our institution, and we have no direct experience with this technique.

In our series, we observed a number of lesions that dis- played endobronchial growth at the onset of disease. Patients who were diagnosed endoscopically to have infiltration of either the carina or the main stem bronchus and in whom the tumor shrunk were treated according to the result of frozen sections. Sleeve pneumonectomy or pneumonectomy were avoided in favor of sleeve lobectomy whenever possible. We have done that in several cases, and we have experienced no local recurrence in the airway so far.

One of the slides showed that, indeed, 34 patients who had $\mathrm{N} 2$ disease at the onset were downstaged to $\mathrm{N} 1$ or $\mathrm{N} 0$ at the operation. This is probably a good result per se, but the survival of these patients is still disappointing.

The issue of postoperative therapy is, as you most correctly pointed out, a controversial one. We started our study in 1990 with the idea of the administering adjuvant therapy to all patients who either did not have a complete resection or who had pathologic N1 and N2 disease. We have found, however, that it is difficult to administer postoperative adjuvant therapy on a regular basis in these patients, because their general conditions might be too compromised to safely allow it. Although we do not have specific data, we have had the impression that postoperative therapy does not appear to influence survival.

Dr Walter Weder (Zurich, Switzerland). What is your operative strategy after neoadjuvant chemotherapy if you have a major response? When do you perform a minor resection, and based on what analysis do you decide?

Dr Rendina. Intraoperative frozen sections are of first importance because the operative field is difficult to interpret macroscopically after induction therapy. We have noticed that instead of being a guide toward a more extended resection, frozen sections have been a guide to restrict and limit the resection. There were many patients in our series in whom either the SVC or the adventitia of the aorta seemed infiltrated by tumor. We then realized by frozen sections that there was no tumor infiltration but simple reactive tissue, which could be left in place. This, of course, also applies to the resection of the bronchus and pulmonary artery.

Dr Carlos Saldarriaga (Medellin, Colombia). Did you do muscle transposition for the bronchial stump in all patients?

Dr Rendina. When we are planning to perform a sleeve resection, which happens as Dr Faber pointed out earlier, when one major bronchus was infiltrated before induction therapy, we take down the intercostal muscle to wrap the anastomosis at the end of the procedure. 